\title{
Croissance brute et pertes par litière de feuilles de Dactylis glomerata (L). Evaluation de conduites de pâturage tournant dans des élevages laitiers du sud-ouest de la France
}

\author{
H Ducrocq, M Duru \\ INRA, Station d'Agronomie, Toulouse, BP 27, 31326 Castanet Tolosan Cedex, France
}

\begin{abstract}
Au printemps, l'intensité et la fréquence de pâturage sont souvent mal adaptées à la quantité d'herbe offerte au troupeau. Les intrants (azote) et l'herbe sont alors mal valorisés. Or pour assurer le maintien d'une production en quantité et qualité suffisantes, il importe de trouver la meilleure combinaison entre intensité, fréquence d'utilisation et fertilisation pour favoriser la production de nouvelles feuilles et limiter les pertes en litière. Nous proposons de modéliser les flux de tissus foliaires et d'utiliser ces modèles pour évaluer des pratiques de pâturage tournant.
\end{abstract}

Un modèle élaboré en domaine expérimental (méthode des talles baguées: Davies, 1981, Sward Measurement Handbook, 179-208) permet de calculer le nombre de feuilles apparues entre deux défoliations sur une talle de dactyle. Ce nombre est une fonction de la longueur de feuilles résiduelles, de l'intervalle entre deux défoliations (cumul des températures moyennes journalières en degrés jour : dj), et d'un indice de nutrition azotée (fonction de la quantité de biomasse et de sa teneur en azote - Duru, 1992, Agronomie, 12, 219-33). L'état du couvert prairial de 12 parcelles ( 3 élevages) est caractérisé, pour chaque Sortie (S) et chaque Entrée $(E)$ de pâturage, par la structure de talles de dactyle ( $n=40$ par observation). Le flux des tissus foliaires (croissance brute et pertes par litière) entre deux défoliations est déterminé à partir du modèle précédant en déduisant les longueurs des différentes feuilles de la talle $\mathrm{S}$, de celles de la talle $\mathrm{E}$.

La caractérisation du couvert végétal avant et après un pâturage montre une grande variabilité pour les critères mesurés (distribution quasi-normale). Ainsi la longueur de feuilles résiduelles varie de 11 à $50 \mathrm{~cm}$, la durée entre 2 défoliations de $110 \mathrm{dj}$ à $500 \mathrm{dj}$, et les indices de nutrition azotée de 65 à 105. La Croissance Brute (CB), résultant de l'apparition et de l'élongation de feuilles varie selon les repousses de 7 à $37 \mathrm{~mm} / \mathrm{jour}$; les pertes de feuilles par Litière de 1,3 à $22 \mathrm{~mm} / \mathrm{jour}$. La proportion de Lit représente ainsi 7 à $80 \%$ de CB. Elle est la plus faible $(<25 \%)$ pour les Lit les plus faibles (< à $0,6 \mathrm{~mm} / \mathrm{jour}$ ) soit un tiers des observations et est importante (de 50 à $80 \%$ ) pour les CB les plus réduites ( $<$ à 1,2 $\mathrm{mm} /$ jour). Le dernier tiers correspond à des Lit et $\mathrm{CB}$ moyennes. Par des régressions multiples pas à pas $(\alpha=0,05), \mathrm{CB}$ et Lit sont mis en relation avec des variables caractérisant le couvert prairial et des variables climatiques. On s'affranchit de l'effet 'temps de repousse' en étudiant $C B$ et Lit par jour. On trouve que $(n=45): C B / j=-2,4+0,2 \times$ $\mathrm{Tm}+0,03 \times \mathrm{LfvS}+0,01 \times \mathrm{IN}\left(\mathrm{r}^{2}=0,82\right) ;$ Lit $/ \mathbf{j}=0,19+0,04 \times \mathrm{Tm}+0,03 \times$ LfS $-0,01 \times$ IN $\left(\mathrm{r}^{2}=0,65\right)$; où IN est l'indice de nutrition azotée, Tm la température moyenne, LfS la longueur de feuille en $S$ et LfvS la longueur de feuille verte en $\mathrm{S}$.

L'IN dépend en partie des apports d'engrais, mais aussi de la biomasse résiduelle et du temps de repousse. LfvS et LfS sont proportionnelles à l'intensité de pâturage. Ainsi les variables retenues par l'analyse traduisent l'effet des pratiques. La proportion de Lit représente une part d'autant plus importante de CB que LfS est élevée, que ces feuilles sont déjà âgées et que I'IN est faible. De fait les pratiques de pâturage qui conduisent à laisser après la défoliation un capteur photosynthétique suffisamment développé (LfvS) mais pas trop âgé (LfS) permettent les CB les plus élevées et les Lit les plus faibles (intensité ou fréquence élevées). Par contre, celles qui laissent beaucoup de feuilles longues ne permettent qu'une CB faible et des pertes élevées (utilisation et fréquence faibles). Une nutrition en azote faible tend à réduire la $\mathrm{CB}$ et accroître la proportion de Lit (et inversement). $\mathrm{Ce}$ modèle est une base indispensable pour valider un modèle d'accumulation de biomasse en condition de pâturage tournant, afin d'évaluer la quantité et la qualité de l'herbe que peut offrir le pâturage. 